\title{
A Social Network Analysis of a Massively Multi-Player On-Line Role Playing Game
}

\author{
Markus Schatten, Bogdan Okreša Đurić \\ Artificial Intelligence Laboratory \\ Faculty of Organization and Informatics \\ University of Zagreb \\ Pavlinska 2, $42000 \operatorname{Vara}_{i}$ din, Croatia \\ Email: (markus.schatten, dokresa)@foi.hr
}

\begin{abstract}
An instance of a massively on-line role playing game called The Mana World is analyzed for its social networks. The gathered data is the result of the first phase of the ModelMMORPG project in which players played a specially designed quest during a controlled experiment. Observations based on the monitored virtual community behaviour and individual elements of interaction are visualised herein and analysed using social network analysis. Insight into the dynamics of the studied virtual society is given along with observations regarding structure of groups and individuals alike.
\end{abstract}

Keywords - virtual community, MMORPG, social network analysis

\section{INTRODUCTION}

The ModelMMORPG (Large-Scale Multi-Agent Modeling of Massively On-Line Role-Playing Games) project ${ }^{1}$ employs a combined empirical and theoretical approach towards finding organizational patterns in gaming behaviour of both humans and artificial agents. From the empirical side, the project studies the human behavior on a number of venues across various gaming servers in order to find most suitable organizational patterns employed by most successful player communities. From the theoretical side, a multitude of organizational architectures from organization theory in various massively multi-player on-line role playing game (MMORPG) settings is tested, and compared with methods found in empirical research. The project is aimed towards enriching the organizational design methods for the development of MMORPGs and to understand the underlying principles of self-organizing and adaptable networks of large-scale multi-agent systems.

According to Wikipedia massively multi-player on-line game (MMOG) is a (computer) game that supports a great number of players playing on-line simultaneously causing or even fostering interaction among them. Role-playing games (RPGs) are a genre in which the player controls the actions of some protagonist (or potentially several party members) in a world which is well defined. MMORPGs are thus a mixture of these two genres allowing players to control the action of their protagonist (avatar) by interacting with a potentially large user-base on-line.

MMORPGs have a number of different subgenres, but a usual setting is that a protagonist is placed into a world in which he interacts with various NPCs (Non-Playing Characters) and mobs which give out tasks (quests) that it has to solve to be able to buy better equipment, learn new skills like magic and similar, or proceed to higher levels. In ModelMMORPG we have chosen The Mana World (TMW) ${ }^{2}$ MMORPG to conduct our research. The reasons for selection were: (a) it is open source (GPL licensed) allowing us to modify code and add additional functionality, (b) it has a supportive community, (c) it supports a number of interaction techniques which can be studied (e.g. trade among players, IRC based chat, organizing teams called parties, social network functions e.g. friends, enemies, parties etc.), (d) it is a (more or less) finished game featuring lots of quests that can be analyzed.

In ModelMMORPG, we have designed a special quest in which players ought to organize their activities in order to solve it. The quest ${ }^{3}$ has been designed during a 3-day brainstorming session and later on developed by project members [1]. Afterwards, during a data collection phase players (mostly students from three countries) have played the game during a period of one month. Every participating player has been informed about the data gathering process, and due to potential privacy issues, all the collected data has been rendered anonymous. After data collection, the data was analyzed using social network analysis (SNA) techniques in order to find patterns of organizational behavior among successful players. Some initial results of this analyses are presented herein.

The rest of this paper is organized as follows: firstly in section II we give an overview of relevant literature. Then in section III we provide a social network analysis of the various social networks that have formed during the data collextion phase. Afterwards in section IV we discuss our findings and in section $\mathrm{V}$ we draw conclusions and give an outline of future research.

\footnotetext{
${ }^{1}$ See http://ai.foi.hr/modelmmorpg for details.

${ }^{2}$ See http://themanaworld.org for details.

${ }^{3}$ See http://dragon.foi.hr for details.
} 


\section{LITERATURE OVERVIEW}

MMORPGs are an interesting modern resource for analyses in various fields of research due to their numerous users, large market share and various opportunities for individual, group and global activities [1]. Apart from providing a data source with unprecedented quantity of data available, MMORPGs are a source with enhanced ease of access to the collected data, which is a crucial point in modern social sciences [2]. Where social sciences research in the past demanded several weeks of working time only to gather data relevant for a research, using methods that might introduce biases, data gathering using modern digital sources based on virtual societies, such as MMORPGs, introduce fast delivery of relevant data with subject behaving as when they are not watched.

MMORPGs are a source of data unimaginable in the past - gathered comprehensive data can include all the data on every single action each of the participating players ever takes, with much less effort. MMORPGs represent a tool for gaining insight into collective human phenomena and large-scale social dynamics [3][4]. Instead of being exclusively socially oriented, MMORPGs can be used as sources of economic data as well, offering a natural environment that supports conducting of behavioural economics experiments [2]. Contrary to the positive aspects of virtual realities found in games, cyber-behaviour can become twisted, just like real-life societies. For example, Powers arguing about virtual communities as concepts with possible moral wrongs, provided insight on how cyber-bullying, and in extremes cyber-rape, applies to interactions in gaming [5].

Szell and Thurner [2] provide an insight in measuring social dynamics in MMORPGs. Interaction types were discussed by Son et al. in [6], based on six distinct interactivity networks. Research conducted by De Simone [7], shows how good aspects of computer games can have a rather negative aspect on social behaviour of young players. Insightful analysis of players of an MMORPG and their behaviour, based on the names of their in-game player characters was conducted by Thurau and Drachen, in [8, pp. 270-281].

\section{ANALYSIS \& RESULTS}

The analysis of anonymized data offers interesting insights into virtual communities formed in the tested instance of TMW. Interaction and communication observed amongst various players, enriched with existent relations associated with players and parties, suggest a firm basis for player behaviour analysis. The following results are based on data of 181 player characters active in TMW game on the designated server.

Every player can describe their relation with another player using one of six relation types: Be Friend, Disregard, Ignore, Blacklist, Set as Enemy, or Erase. Each of the mentioned relations serves as character socialisation type specification. Character relations were gathered at the end of the testing period and therefore represent only a certain point in time, i.e. at the end of the testing period.

Visualisation of character relations discovered several groups that formed during the data collection period. These communities, shown on Fig. 1 (see appendix), span from three to multiple users.

Character relations are directed though, therefore Fig. 1 shows a directed graph. Vertexes are sized according to their outdegree (number of arrows with the given vertex as a source), allowing for easier tracking of who initiated creation of a relation. If one player is related to another player, the opposite does not necessarily hold. The number of arrows (out-degree of a vertex) shows how much a player depends on other players, i.e. with how many players the given player wants to be in constant contact (easier access to direct messages, information on their position, etc.).

Players can cooperate an exercise teamwork by grouping into avatar (player character) groups. Such groups of avatars are called parties. During the data gathering period, only a couple of parties formed, even though party membership was a prerequisite for successful completion of the designed quest. Two largest parties include nine and seven characters respectively. Party membership is beneficial for avatars since experience (character progression) and loot (items) are divided amongst geographically close party members, making success easier to achieve. It is interesting to note a large number of formed parties, yet a rather small number of members per party, as is visible in Fig. 2 (see appendix).

Members of a certain party are players who are determined to act in good faith and cooperate. As opposed to character relations, party membership is more exclusive and can be considered a privilege. While character relations are one-sided and private, one can become a party member by invitation only, and a party member can be expelled from a party at any given time. Party structure therefore portrays the given community in a more comprehensive way.

Character parties are mainly created by players of similar interest, be it game progression, diverse character traits, character occupation, etc. When creation time of parties is observed against characters by their time of creation, and number of parties created is opposed to the quantity of characters created at a certain time, taking into account several observed massive character creation periods as well, simple interesting trends can be identified: parties were regularly created when there was a rapid growth of globally present characters. This observation can be supported by Son et al. and results presented in [6], stating that, in their research, party invitation was the most prevalent interaction type.

As is usual in MMORPGs, players are able to privately communicate with each other. These messages, called whispers, are seen exclusively by the two involved players, while other types of chat in TMW include: general chat (public visibility and 


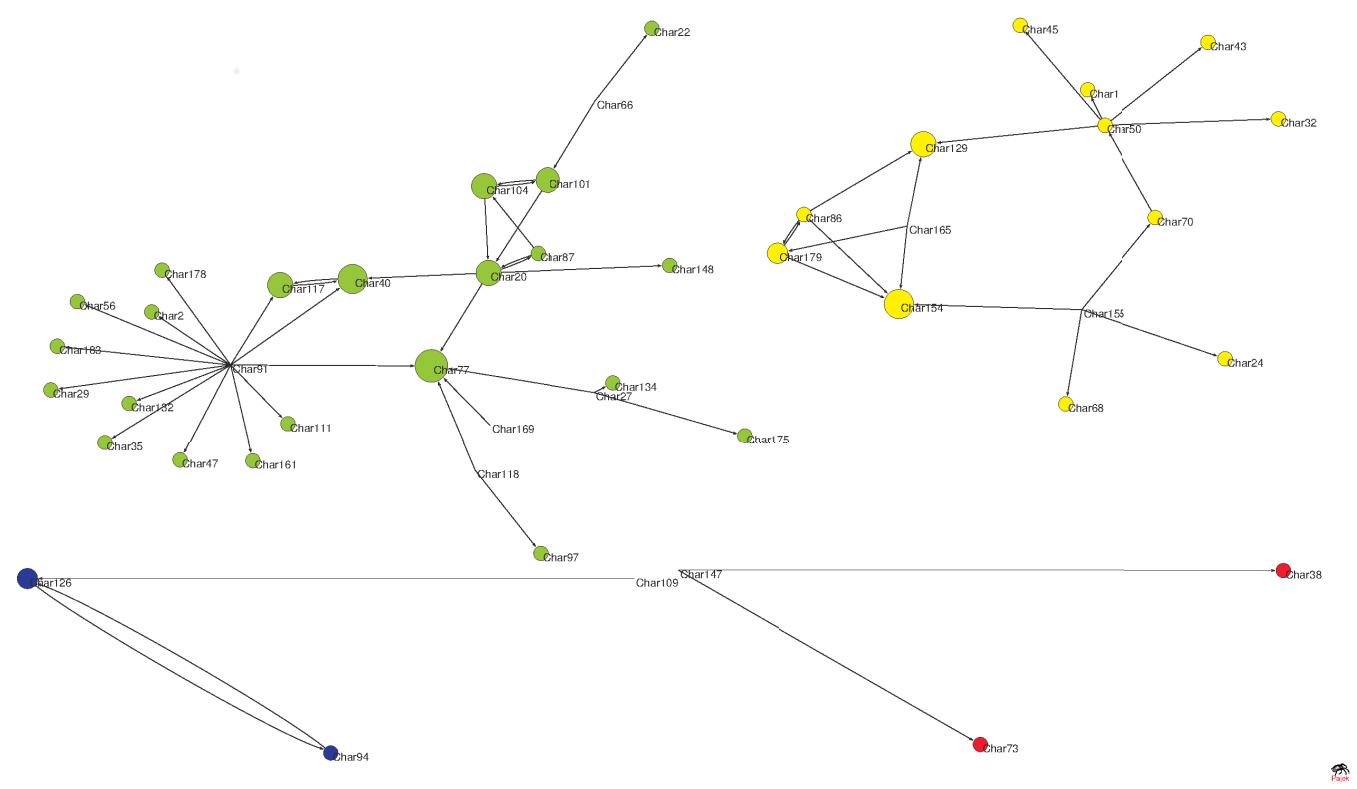

Fig. 1: Visualisation of communities formed during the data collection period, based on character relations, indicates two groups of players most interesting to observe

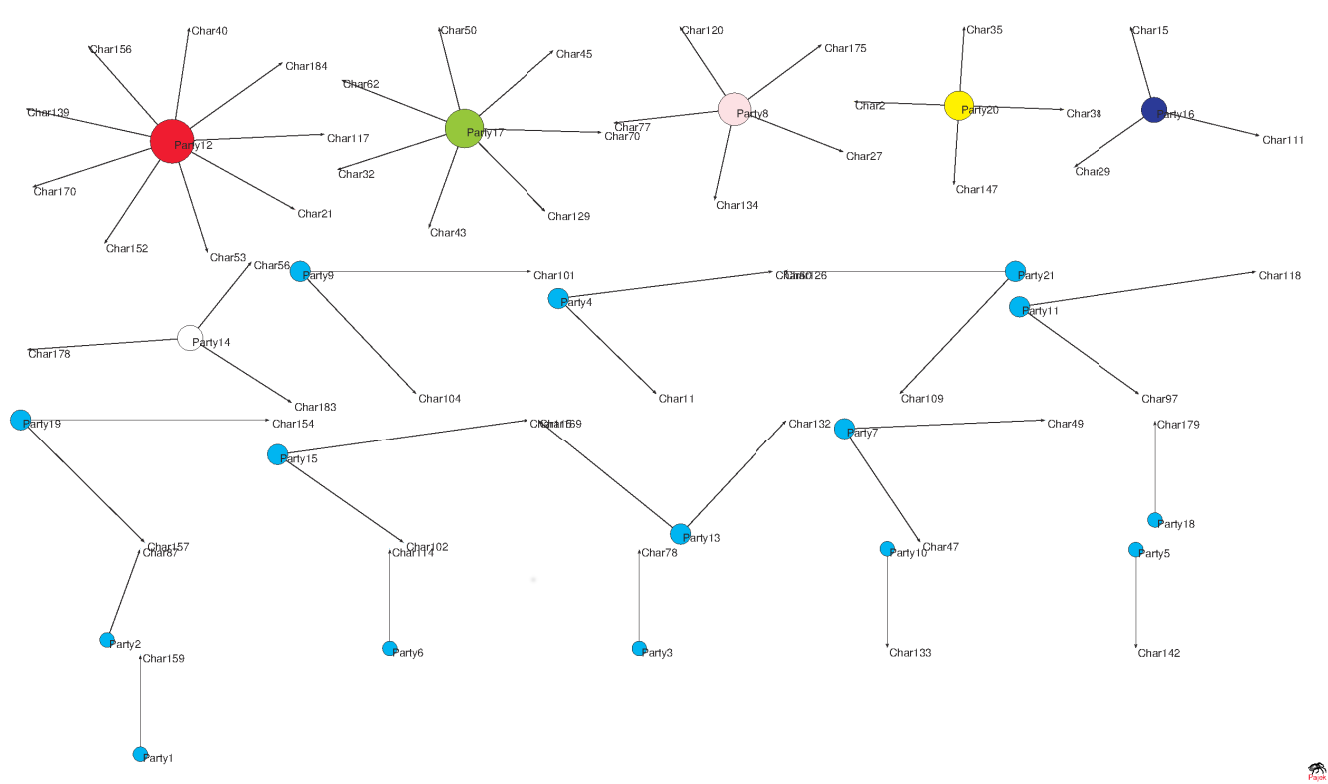

Fig. 2: Visual representation of parties and their members, at the end of the data gathering period

participation), party chat (party-wide visibility and participation), and trade chat (global chat containing preferably trade-related messages). Whispering was the most actively used chat type during the data gathering period. With visually represented whisper chat intensity over time (see figure Fig. 3 in appendix), one can observe periods of community activity.

It is clearly visible from Fig. 3 that the community was active in bursts. This observation can be indicative of the nature of players owning in-game characters - whisper bursts correspond to the measured time (in minutes from the beginning of the data gathering period), i.e. whisper bursts occur in almost regular intervals of 24 hours. This, in turn, might indicate that most of the players are from the same, or very similar, real-life geographical location.

Analysing whisper chat messages, one might conclude that the community was alive during the whole data gathering period. Clearly, there was perceived activity cessation for a period of time - most likely a real-life event prevented players from 


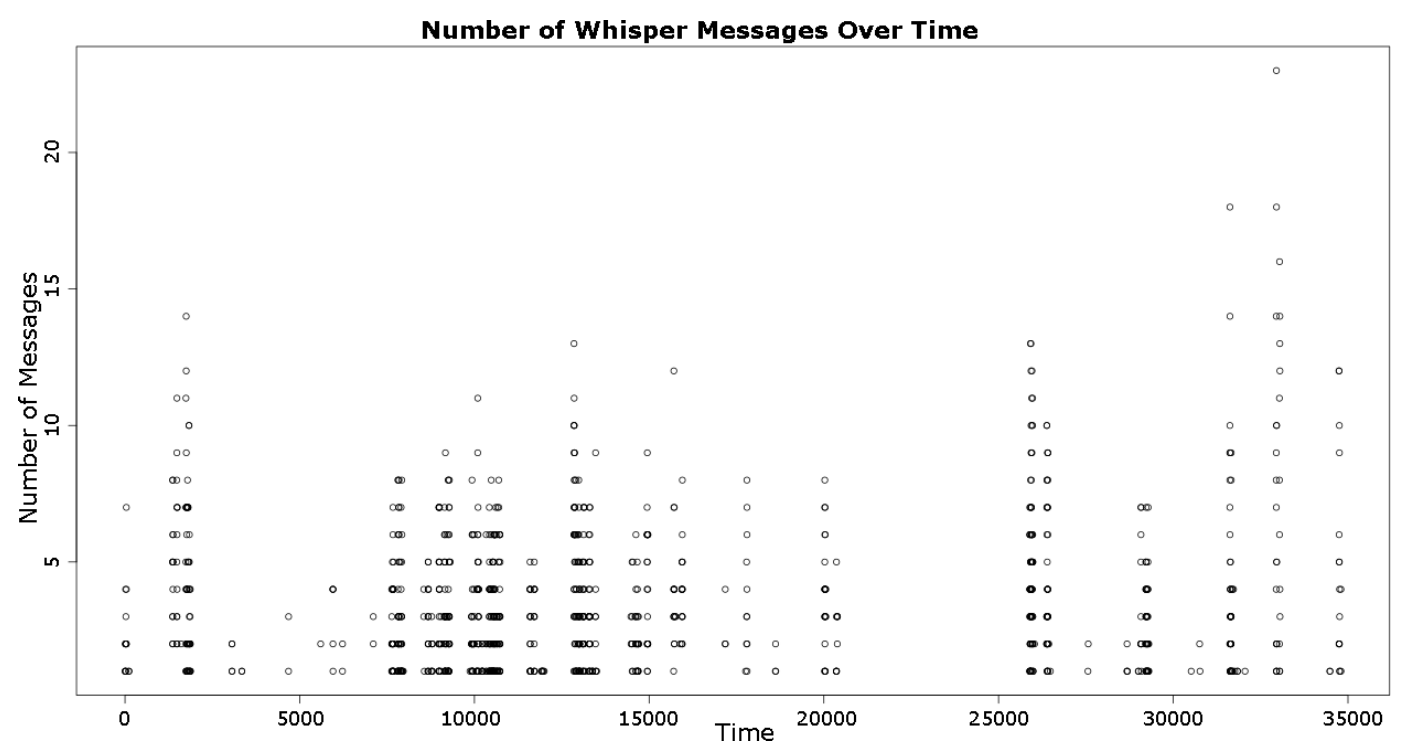

Fig. 3: Quantity of whisper chat messages by time of transmission

participating in the game (this conclusion can be backed up by the fact that most of the players were students and that there was a week reserved for mid-term exams during the data gathering period).

Based on data gathered from whisper chat, analysed in terms of time of the first sent message, and time of the last sent message, for each character, one might draw a conclusion that the community did not consist of players who were interested in the game for a prolonged period of time. Analysis shows that only a few characters stayed active after early inclusion in the game. Furthermore, only a few characters active during the first half of the data gathering period remained active in the game throughout the whole period, and most of characters showed signs of activity for a short period of time only. This might indicate that the community mostly consisted of characters of rather short life- or activity-span.

As mentioned earlier, one type of chat available in TMW is party chat. Available only to members of a certain party, party chat bears witness to formation of micro-communities of a kind - the character parties. A party chat can be accessed, for consumption or participation, by all the members of a given party. Active party chat indicates a party of active members, due to either social, organisational, or another kind of behaviour, or their combination.

Figure 4 (see appendix) contains a visualisation of the quantity of party chat messages, and their frequency is somewhat similar to the distribution observable in the frequency of whisper messages, where clearly distinct bursts of party chat usage are visible. Based on the data on the frequency and quantity of exchanged party messages, versus the frequency and quantity of whisper chat usage, one could observe that micro-communities are more lively. Since teamwork and party membership were prerequisites for the designed quest, peaks in party chat quantity might indicate that users were preparing strategies for finishing the designated quest.

\section{DISCUSSION}

Virtual community of 181 people who do not necessarily act according to their real-life personality traits, during the data gathering period of roughly 25 days provided us with a large amount of data and interesting analysis opportunities.

Virtual community formed in TMW was not built without purpose. All the players had a shared goal - finishing a quest designed especially for this research. The assumption that most of the players played the game with sole goal of finishing the mentioned quest might affect the results and gathered data. The quest demanded cooperation, competition, and forming of alliances though, therefore propagating such values. This, and further research, are the main reasons why analysis is largely concerned with the mentioned values.

Virtual communities, as long as being controlled by human users, i.e. representing human users in a virtual environment, are prone to be influenced by real-life stimuli. This assumption can be best observed in available data on party and whisper chat frequencies: time period between 20.000 and 25.000 time units is rather unpopulated, i.e. activity is almost non-existent. The mentioned time period corresponds to the real-life event of mid-term exams.

Analysis of whisper chat data, according to senders and receivers of a specific message, ignoring time constraints, provides one with seemingly the most influential individual in the community. Should a community member be evaluated by the amount of messages they send to their environment, Fig. 5 detects the most active members of the community, based on whisper message exchange, and all the included player characters. 


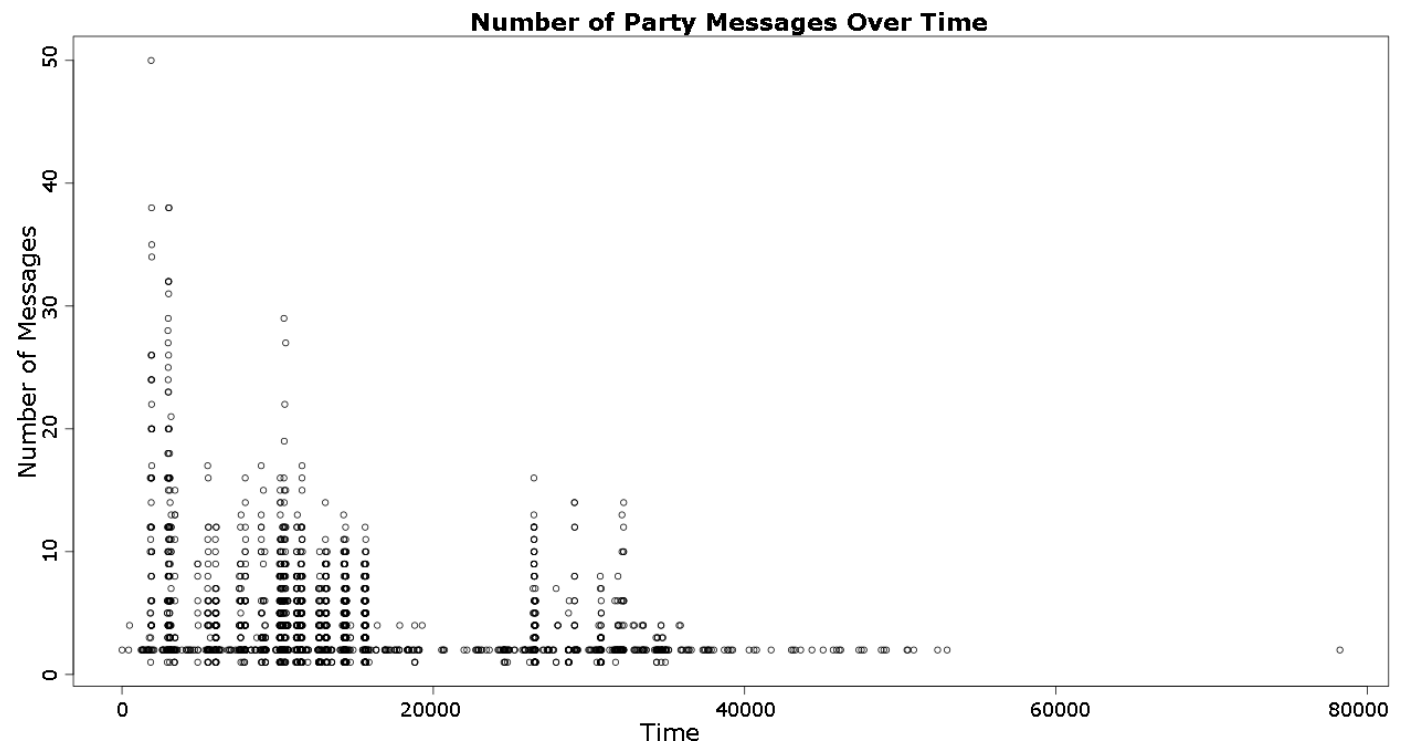

Fig. 4: Quantity of party chat messages by time of transmission

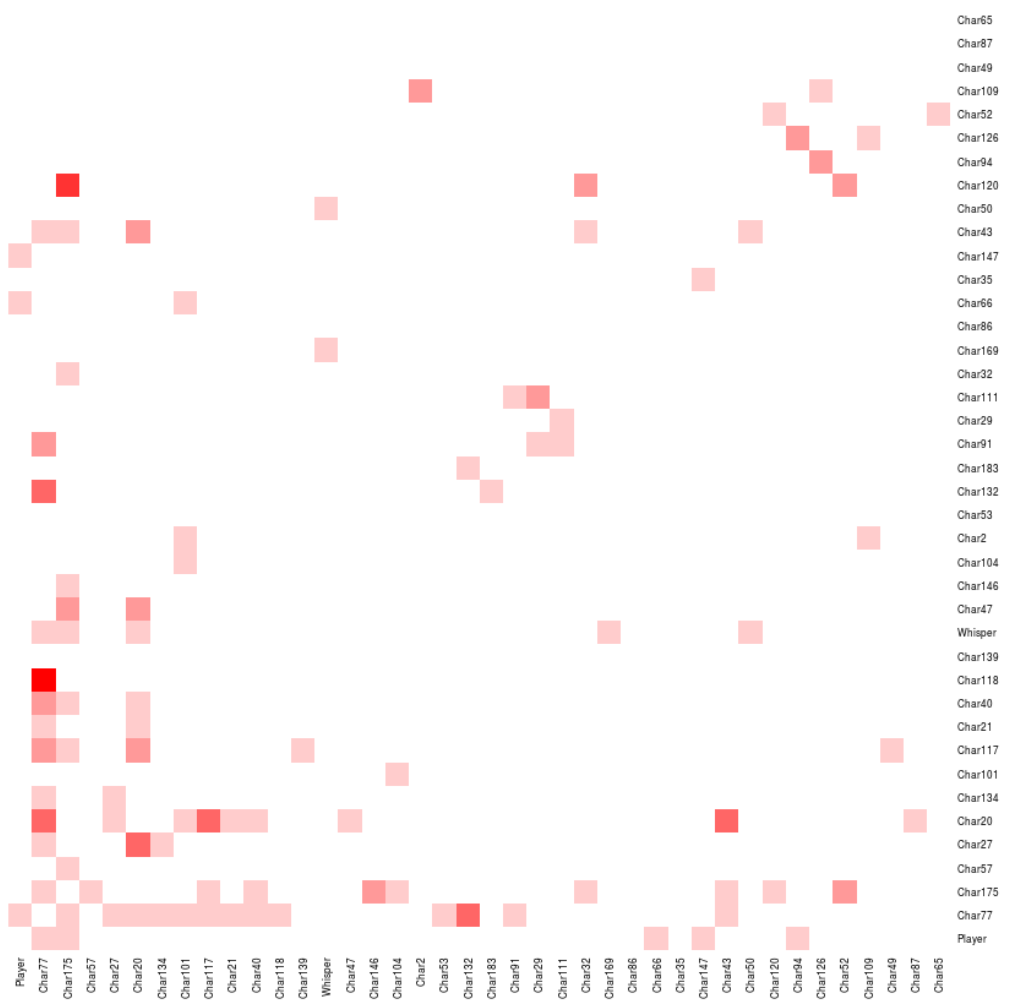

Fig. 5: Heatmap-styled visualization of intensity of whisper chat message exchange where vivid red rectangles depict high intensity

\section{CONCLUSION}

With continuous growth, and increasingly large number of players, MMORPGs represent a vast playground for virtual society and virtual communities [1]. In a process similar to any computer-based activity, almost any activity in an MMORPG produces data, which is stored in order for the game to process smoothly. Such data can be used in various ways, from spying to social 
analyses.

Data analysis described in this paper uncovered several potentially interesting online community concepts: it is shown that real-life activities have heavy impact on the virtual community (although virtual environment can have a negative effect on players and their real-life performance [9]).

Formed virtual communities were mostly observed according to whisper chat frequency, party chat frequency, character relations and party membership. The observed behaviour may be indicative of the way members of short lived virtual societies act in an environment, when mostly driven by a specific task.

Open questions that remain unanswered herein are related to the dynamics of social networks and likewise community formation during time. How did the various networks form? What were the basic activities that led towards network formations? How are these activities related to the communication that was conducted between the actors? Can we model these activities using agent based simulation? These and similar questions are subject to our future research.

\section{ACKNOWLEDGMENT}

This work has been supported in full by the Croatian Science Foundation under the project number 8537.

\section{REFERENCES}

[1] M. Schatten, I. Tomičić, and B. Okreša ÄŘurić, "Multi-agent Modeling Methods for Massivley Multi-Player On-Line Role-Playing Games," in MIPRO, Opatija, HR, 2015

[2] M. Szell and S. Thurner, "Measuring social dynamics in a massive multiplayer online game," Social Networks, vol. 32, no. 4, pp. 313-329, 2010. [Online]. Available: http://dx.doi.org/10.1016/j.socnet.2010.06.001

[3] W. S. Bainbridge, "The scientific research potential of virtual worlds." Science (New York, N.Y.), vol. 317, no. 5837, pp. 472-6, 2007. [Online]. Available: http://www.ncbi.nlm.nih.gov/pubmed/17656715

[4] E. Castronova, "On the Research Value of Large Games: Natural Experiments in Norrath and Camelot," Games and Culture, vol. 1, no. 2, pp. 163-186, apr 2006. [Online]. Available: http://gac.sagepub.com/cgi/doi/10.1177/1555412006286686

[5] T. M. Powers, "Real wrongs in virtual communities," Ethics and Information Technology, vol. 5, no. 4, pp. 191-198, 2003. [Online]. Available: http://www.springerlink.com/openurl.asp?id=doi:10.1023/B:ETIN.0000017737.56971.20

[6] S. Son, A. R. Kang, H.-c. Kim, T. T. Kwon, J. Park, and H. K. Kim, "Multi-relational social networks in a large-scale MMORPG," p. 414, 2011. [Online]. Available: http://conferences.sigcomm.org/sigcomm/2011/papers/sigcomm/p414.pdfhttp://dl.acm.org/citation.cfm?doid=2043164.2018496

[7] J. J. De Simone, "What Is Good Can Also Be Bad: The Prosocial and Antisocial In-Game Behaviors of Young Video Game Players." Atlantic Journal of Communication, vol. 21, no. 3, pp. 149-163, 2013. [Online]. Available: 10.1080/15456870.2013.801756\$backslash\$nhttp: //ezproxy.umsl.edu/login?url=http://search.ebscohost.com/login.aspx?direct=true $\{\backslash \&\} \mathrm{db}=$ aph $\{\backslash \&\}$ AN=89456680 $\{\backslash \&\}$ site $=$ ehost-live $\{\backslash \&\}$ scope $=$ site

[8] J. Anacleto, S. Fels, N. Graham, B. Kapralos, M. S. El-Nasr, and K. Stanley, Entertainment Computing âĂŞ ICEC 2011, ser. Lecture Notes in Computer Science, J. C. Anacleto, S. Fels, N. Graham, B. Kapralos, M. Saif El-Nasr, and K. Stanley, Eds. Berlin, Heidelberg: Springer Berlin Heidelberg, 2011, vol. 6972. [Online]. Available: http://link.springer.com/10.1007/978-3-642-24500-8

[9] C. S. Peters and L. A. Malesky, "Problematic usage among highly-engaged players of massively multiplayer online role playing games." Cyberpsychology \& behavior : the impact of the Internet, multimedia and virtual reality on behavior and society, vol. 11, no. 4, pp. 481-484, 2008. 